\title{
Pathology of five Scottish deaths from pneumonic illnesses acquired in Spain due to Legionnaires' disease agent
}

\author{
J. F. BOYD ${ }^{1}$, W. M. BUCHANAN ${ }^{2}$, T. I. F. MacLEOD ${ }^{3}$, R. I. SHAW DUNN 4 , \\ AND W. P. WEIR ${ }^{5}$ \\ From ${ }^{1}$ Brownlee Laboratory, Ruchill Hospital, G20 9NB and University Department of Pathology, \\ Western Infirmary, Glasgow G11 6NT, 2 Pathology Department, Stobhill General Hospital, Glasgow G21 \\ $3 U W,{ }^{3}$ Pathology Department, Western Infirmary, Glasgow G11 6NT, ${ }^{4}$ Pathology Department, Law \\ Hospital, Carluke, Lanarkshire ML8 5ER, and ${ }^{5}$ Pathology Department, Royal Alexandra Infirmary, \\ Paisley, Renfrewshire PA2 6LX, UK
}

SUMMARY The pathology and histology are reported of five Scots who died of severe pneumonic illnesses after holidays in Spain, three in 1973 and two in 1977. There is strong evidence in favour of all the deaths having been due to the newly discovered Legionnaires' disease (LD) agent. The agent (or its soluble antigen) has been visualised in sections of lung tissue by fluorescent-antibody tests in all cases, and the agent has been identified by the Dieterle silver staining method in small numbers in all cases. Serological testing was possible in three of the patients, and two had very high antibody titres against the LD agent.

Apart from the extensive and severe nature of the pathological process there is no feature to distinguish pulmonary infection by this agent from that due to more commonly known bacteria capable of causing lobar pneumonia. The severity and extensive nature of the process is partly a reflection of neglect in seeking treatment until late in the infection, and partly a reflection, as revealed in retrospect, on the use of the wrong antibiotic combination during treatment.

Erythromycin has been recommended by other workers as the drug of choice against the LD agent. Infection by this organism is not confined to the USA or to Spain and is indigenous also in the United Kingdom.

At the end of July 1973, 252 Scottish tourists returned to Glasgow after spending a holiday in Spain. One hundred and sixty-four of the party, who had all stayed in the same hotel, had been ill with alimentary and respiratory tract complaints; one died in the aeroplane during the return flight. Nine were admitted to hospitals in and around Glasgow during the next few days, and two died. This episode and the ensuing studies have been reported recently (Lawson et al., 1977; Reid et al., 1978).

Almost four years later, another patient was admitted to one of our hospitals with a pneumonic illness after a holiday in the same Spanish hotel, and this patient died. One month later, a fifth patient was admitted to another hospital, having become

Received for publication 12 April 1978 ill after returning from holiday in another part of Spain, and he died.

Meanwhile a large outbreak of severe respiratory illness affected 180 of around 4400 persons attending the Annual Convention of American Legionnaires in Philadelphia, USA, in July 1976, and there were 29 deaths (Fraser et al., 1977). Intensive investigation of this event by the Americans yielded a hitherto unknown Gram-negative agent (McDade et al., 1977), which has been named temporarily Legionnaires' disease agent (LD agent). Preliminary reports about this organism were circulated in the early months of 1977 , and, with the fourth death occurring locally, an effort to link the three episodes on two continents achieved success, and later the fifth death was shown to have a similar aetiology.

This article reports the relevant gross and histological features of the five patients who died in the west of Scotland. 


\section{Clinical summary, necropsy findings, and histology}

The findings are summarised in the Table.

PATIENT 1 (male, aged 54)

This man's illness presented initially with vomiting and diarrhoea, and later he became weak and mentally confused. Breathlessness developed later, and he died on the return flight home. There was a past history of chronic bronchitis and obesity. Postmortem examination showed left lower lobe lobar pneumonia. Histology (Fig. 1) showed

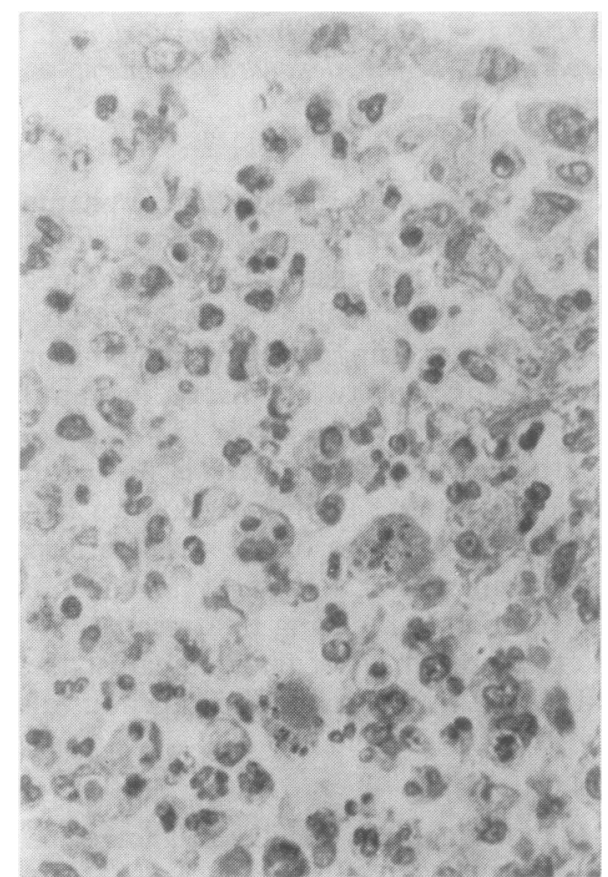

Fig. 1 Patient 1. Pneumonic area showing neutrophil polymorphs mixed with mononuclear cells, several bearing carbon pigment. Scanty red blood corpuscles. No fibrin in this area. Prominent alveolar epithelium above. Haematoxylin and eosin $\times 600$. neutrophil polymorphs and macrophages in roughly equal numbers. Alveolar fibrin and oedema were patchy. Alveolar hyperplasia suggested a reparative phenomenon. There was minimal interstitial oedema. The bronchioles showed no damage, and mixed thrombi affected some smaller blood vessels, but extensive intravascular coagulation was absent.

An incidental finding was a small renal carcinoma.

PATIENT 2 (male, aged 62)

This man developed fever, followed by diarrhoea, four days before returning home. He was very breathless and cyanosed when he reached home and he entered hospital two days later but died on the same day. In the past he had had chronic bronchitis and pernicious anaemia. At necropsy the trachea appeared to be inflamed. The lungs ( $1476 \mathrm{~g}$, L $1645 \mathrm{~g}$ ) showed recent fibrinous pleurisy, more marked on the left side. Dissection showed grey hepatisation of the upper third and lower third of each lung, and the middle thirds were free from involvement. Histology showed lobar pneumonia at the stage of incipient resolution and repair over many areas, but there were foci proceeding to incipient abscess formation. Neutrophil polymorphs and macrophages were present in roughly equal numbers (Fig. 2). In places, lymphocytes were present in small numbers. There was focal alveolar cell hyperplasia, some alveolar haemorrhage, and moderate alveolar oedema. Scanty arterioles contained antemortem thrombi, but there was no vasculitis.

Incidental findings included an old right inferior frontal cerebral infarction and fatty change of the liver.

PATIENT 3 (male, aged 50)

This man developed diarrhoea, fever, and weakness four days before the end of his holiday. After returning home he developed cough and breathlessness and was admitted to hospital three days later. Pneumonia was diagnosed but the blood pressure dropped, and, in spite of intensive therapy, his condition deteriorated relentlessly, death occurring

Table Summary of five patients who died in the west of Scotland from Legionnaires' disease

\begin{tabular}{lllllllll}
\hline Patient & $\begin{array}{l}\text { Year of } \\
\text { death }\end{array}$ & $\begin{array}{l}\text { Place } \\
\text { visited }\end{array}$ & Sex & $\begin{array}{l}\text { Age } \\
\text { (years) }\end{array}$ & $\begin{array}{l}\text { Duration of No. of consoli- } \\
\text { illness } \\
\text { (days) }\end{array}$ & $\begin{array}{l}\text { Serum fuorescent- } \\
\text { dated lobes } \\
\text { agent* }\end{array}$ & $\begin{array}{l}\text { Direct fluorescent- } \\
\text { antibody staining of }\end{array}$ & $\begin{array}{l}\text { Modified Dieterle } \\
\text { staining method }\end{array}$ \\
\hline lung sectionst
\end{tabular}

* Performed by CDC, Atlanta, Ga, USA. ND = not done. Significant titres considered to be $>128$.

†Performed by CDC, Atlanta, Ga, USA, either on paraffin sections of lung (all cases) or on smears made from squeezing formalin-fixed lung patient 4). Only 'soluble antigen' seen in patients 1-3. LD agent seen in patients 4 and 5. 


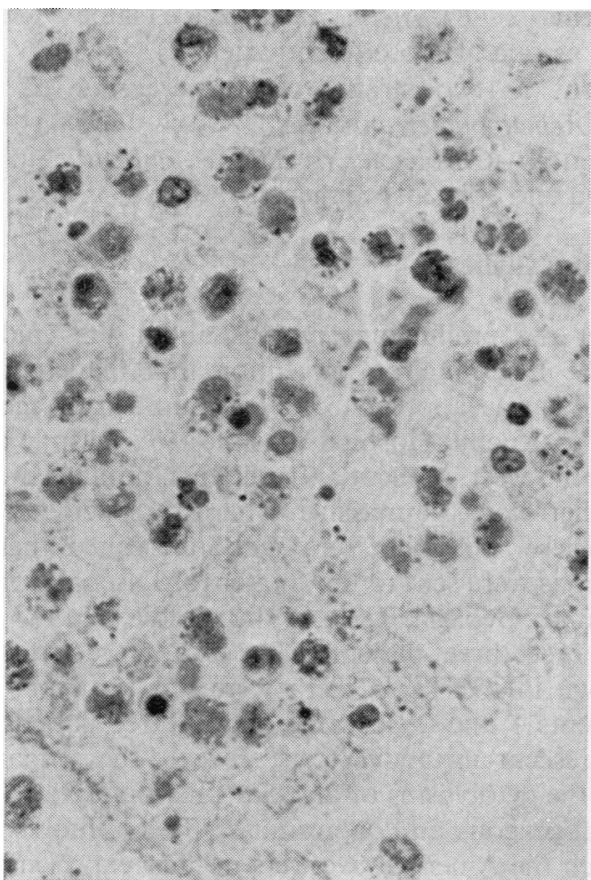

Fig. 2 Patient 2. Pneumonic area. Neutrophil polymorphs and macrophages are both present and contain carbon pigment. Protein-rich fluid with wisps of fibrin. $H$ and $E \times 600$.

five days later. He had diabetes mellitus controlled well by diet and an oral hypoglycaemic agent. One month before going on holiday he had had a pneumonia-like illness, which responded to antibiotic therapy. Postmortem examination showed almost total consolidation of the right lung $(1400 \mathrm{~g})$ apart from the anterior portion of the upper lobe. The consolidated area was unduly oedematous and rather friable. Histological examination showed lobar pneumonia. The alveolar exudate was less cellular than expected (Fig. 3), and mononuclear cells were more abundant than neutrophil polymorphs. Occasional foci showed incipient necrosis and suppuration. Evidence of repair was minimal and there was no evidence of resolution or of organisation. Very scanty asbestos bodies were present. There was a patchy low-grade vasculitis, mostly phlebitis. The left lung ( $595 \mathrm{~g}$ ) was not affected but showed a calcified focus of old tuberculosis at the apex of the lower lobe.

Other findings included toxic megacolon and mild fatty change of the liver.

PATIENT 4 (female, aged 51)

This woman became ill in the same hotel four years after the first three. She felt feverish two days before the end of her holiday. After returning home she developed breathlessness and had four to five loose motions per day. She was admitted to hospital with pneumonia five days later. Her condition deteriorated relentlessly and she died two days later. In the past she had had successful treatment for pulmonary tuberculosis. Postmortem examination showed total consolidation of the right lung (1580 g) at the stage of grey hepatisation with foci of early suppuration in all lobes but especially at the base. The left lung $(860 \mathrm{~g})$ showed consolidation of the upper lobe, excepting the lingula, and fewer foci of suppuration were present there. The lower lobe showed moderate congestion and oedema. Apart from the areas where suppuration was evolving, histology showed that resolution (Fig. 4) and mild organisation were well-established, but the findings in the middle lobe were more in keeping with secondary bronchopneumonia.

Other findings included healed tuberculous foci in the apices of both lungs and a terminal shallow subdural haemorrhage.

PATIENT 5 (male, aged 64)

This man had no connection with the other patients

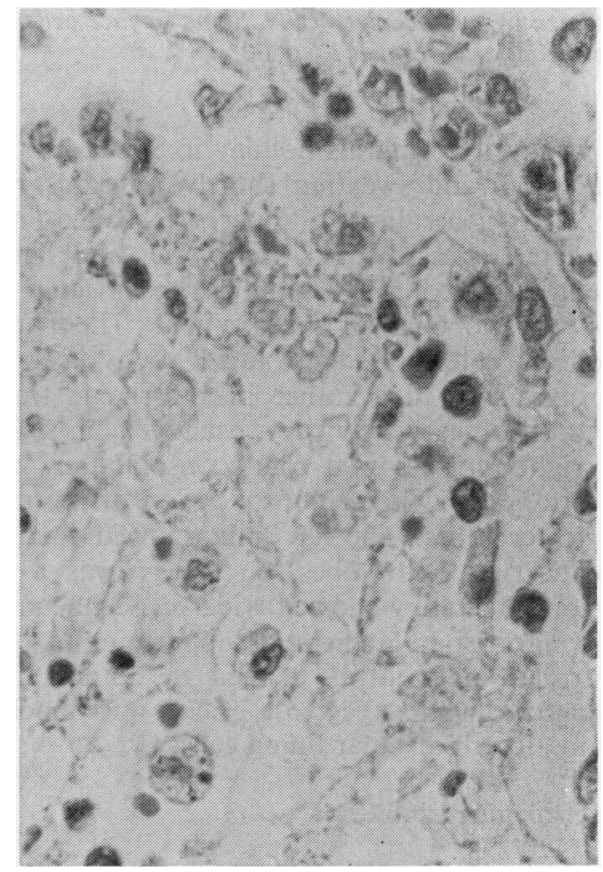

Fig. 3 Patient 3. Pneumonic area rich in fibrin. Cellular exudate more sparse. Foamy macrophages predominate here, neutrophil polymorphs being more abundant elswhere. $H$ and $E \times 600$. 


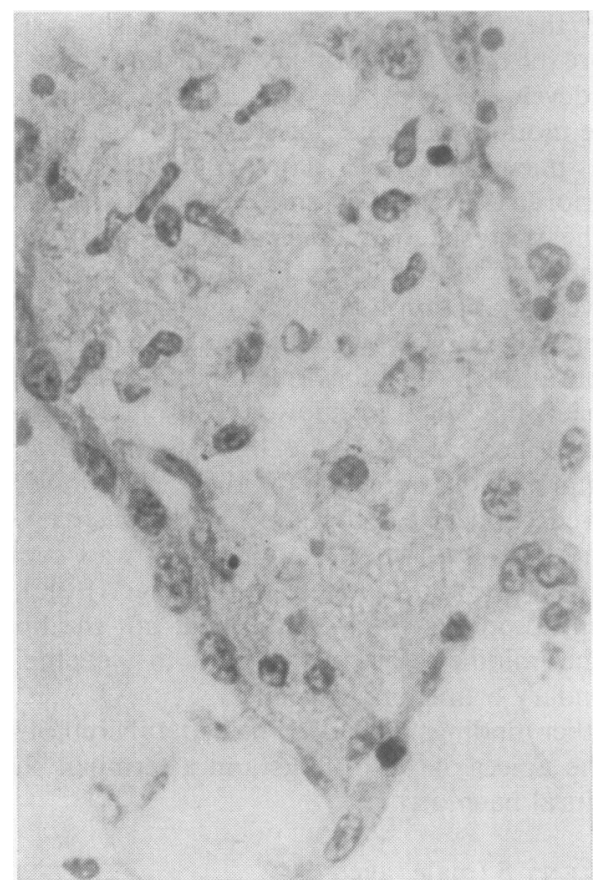

Fig. 4 Patient 4. Degree of resolution shown. Conglomerate rather amorphous fibrin mass covered and permeated by mononuclear cells. Some surface cells may be epithelial cells. $H$ and $E \times 600$.

and was well during his holiday in Spain. He developed rigors and sweating shortly after his return home. His general practitioner was called in five days later, prescribed an antibiotic, and had the patient admitted to hospital 24 hours later when there was no clinical improvement. The patient's condition deteriorated to death two days later. Necropsy showed a rather haemorrhagic trachea, and both lungs were heavy, each weighing $1200 \mathrm{~g}$. The right lung showed total consolidation of the upper lobe and partial consolidation of the middle and lower lobes. The left lung showed total consolidation of both lobes, and there was a small effusion. Histology showed widespread pneumonia, resolving in some areas and suppurating in others. Neutrophil polymorphs tended to dominate over mononuclear cells (Fig. 5). There was much desquamation of alveolar lining cells.

\section{Bacteriological studies}

Specimens for conventional bacteriological studies were taken from the lungs of the first four patients with the following results:

Patient 1-Escherichia coli
Patient 2-Klebsiella aerogenes (same Klebcin type as one of those from patient 3)

Patient 3-two strains of $K$. aerogenes, E. coli, and Acinetobacter anitratus

Patient 4 -Citrobacter freundii, $A$. anitratus (in both blood and spleen specimens).

\section{Further studies}

After the preliminary reports about the epidemic at the Legionnaire Convention in Philadelphia in 1976 , it was appreciated by a number of medical staff in Ruchill Hospital that the 1973 episode affecting the west of Scotland patients mimicked on a smaller scale the massive American experience.

Where possible, sera and unstained paraffin sections were sent to the Center for Disease Control, Atlanta, Georgia, USA in the middle of 1977 from the first three patients. The results reported are shown in the Table. Sera were not available from patients 1 and 2 . In none of the three patients did fluorescent-antibody tests reveal the organism, but the cytoplasms of alveolar macrophages showed homogeneous and/or granular specific fluorescence, which the American workers in their experience had

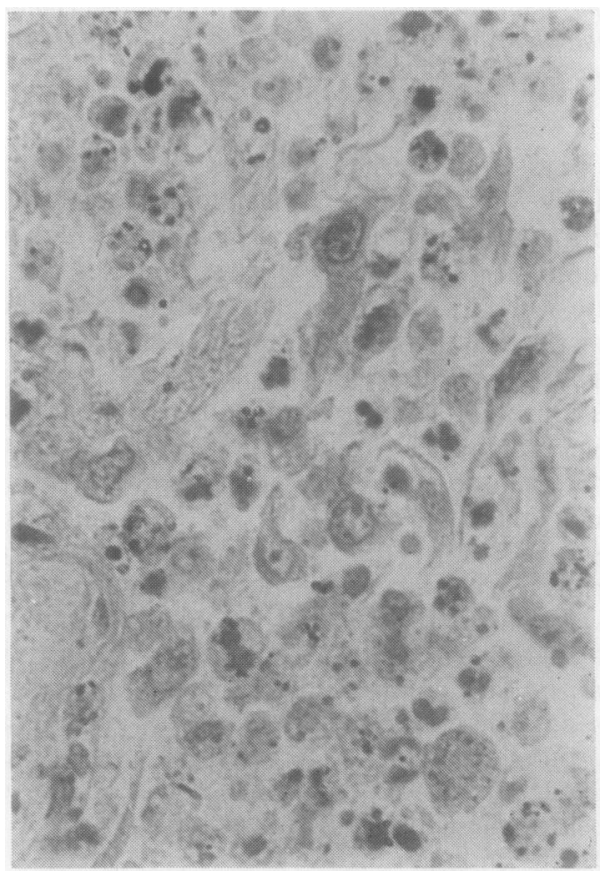

Fig. 5 Patient 5. Area of consolidation with mixture of neutrophil polymorphs, mononuclear cells, and rather bizarre desquamated epithelial cells. Carbon and haemosiderin pigments evident. $H$ and $E \times 600$. 


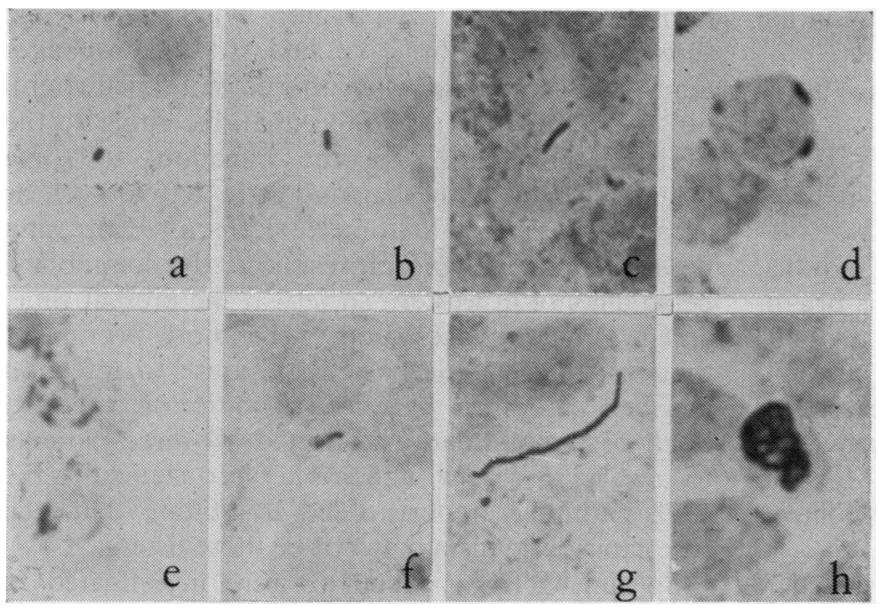

Fig. 6 Various forms of $L D$ agent :

(a) coccobacillus extracellular,

(b) diplococcobacillus extracellular, (c) diplobacillus, a notch being evident at mid-point on inferior edge. Extracellular: (d) two bacilli at different angles on surface of a degenerate cell, $(e)$ intracellular coccobacilli and bacilli, none in perfect focus, $(f)$ intracellular beaded form, two right-hand beads in focus, $(g)$ extracellular filamentous form with tapering ends, very rare, $(h)$ intracellular coiled filamentous form appreciated best on focusing up and down. Dieterle stain. $\times 1500$.

seen only in accompaniment with LD agent infection, and this appearance was interpreted as being due to 'soluble antigen' of the LD agent. In retrospect it is believed that part of the failure to identify the LD agent by fluorescent-antibody tests in patients 1 and 2 might have been due to the use of mercurycontaining fixatives in 1973.

In the case of patient 4 , sera, sections, and a block of formalin-fixed wet pneumonic lung were sent; the results are shown in the Table. An organism with the size and morphology of the LD agent was seen in this instance by fluorescent-antibody tests.

Although patient 5 failed to show an antibody response, a few organisms similar to $L D$ agent were seen in fluorescent-antibody stained sections.

Having received these results from $\mathrm{CDC}$, together with additional features about the organism, it became necessary to try to show the organism in the tissues by the modified Dieterle staining method (Dieterle, 1927; Chandler et al., 1977; van Orden and Greer, 1977).

A number of remarks are necessary about the modified Deiterle staining method. Positive and negative controls are necessary with each batch of slides being stained. Each pathology department might need to make minor modifications to the method to suit its local circumstances. As originally devised, it is a method for staining spirochaetes in formalin-fixed, paraffin-embedded tissues, and it accomplishes this beautifully. In addition to staining the LD agent, the Dieterle method also stains Calymmatobacterium granulomatosis (Donovania granulomatosis) of granuloma inguinale (Blackmon, 1977, personal communication; Chandler et al., 1977; van Orden and Greer, 1977). van Orden and Greer point out that formalin pigment and foreign material in macrophages also stain positively. Therefore, between the presence of dust deposits in the lungs and in lung macrophages, the abovementioned argyrophilic foreign material in macrophages, and formalin pigment (unless efforts are made to remove this before the main staining procedure), there is much in lungs that will be confused with the LD agent until experience has been gained with the size and shape of the agent (Fig. 6).

In none of our cases was the organism as abundant in the lungs as has been illustrated previously (Chandler et al., 1977). The LD agent was always sparse, but, after one organism had been found, others tended to be found singly, usually in the same or adjacent alveoli. While the coccobacillary form is the most common one by far, it is the opinion of JFB that in human lungs the LD agent shows a varied morphology from coccobacillary to bacillary and possibly even filamentous, as has been reported in culture (McDade et al., 1977). It may be seen either extracellularly or intracellularly and, further, it appears to have been seen by JFB in the blood in vessels in the lung, thus suggesting that it can cause septicaemia, as suggested by Macrae (1978).

It is not going to be easy to identify the LD agent in sputa by the Dieterle method, as has been suggested by Macrae and Lewis (1977), and fluorescent-antibody procedures will be much more suitable for these situations.

Further, it seems to JFB that the numbers of LD agent in the lungs of patients $1-4$ are about $25 \%$ of the total population of organisms as revealed by Giemsa's stain, whereas, few though they are, they appear to be the only organism to be identified in the lungs of patient 5 . Although patient 5 shows no antibody response, organisms do not appear to be 
any more numerous or fewer in his lungs than in the lungs of the other four patients.

We have had no opportunity to make ultrastructural studies of any of the lungs.

\section{Discussion}

The LD agent has been recognised for 20 months, and it continues to amaze everyone that this organism, which is moderate-sized (about $3 \times 0.4 \mu$ ) (Chandler et al., 1977; McDade et al., 1977), should have remained unknown for almost 100 years after the end of the main flood of microbiology discoveries, and full credit must be accorded to Drs J. E. McDade and C. C. Shepard at the Center for Disease Control, Atlanta.

The clinical findings reported in this article have been kept brief, and additional laboratory data available during life have not been quoted, to allow attention to be focused on the pathological findings at necropsy and on the histology. A detailed clinical report about patients 3 and 4 has been prepared (Lawson, 1978). The five patients had been ill for 8 , $8,13,10$, and 8 days, respectively, and had received variable amounts of professional and amateur medical attention when in Spain, before death on the aeroplane and also at home before admission to hospital. None of this is detailed.

As discussed by Reid et al. (1978), many possible explanations for the 1973 episode were considered, although it was striking that all three deaths involved men. At that time the pathology of these three cases was assessed by JFB, who was unable to find a common infecting agent in the tissue sections. Although $K$. aerogenes seldom causes fulminant disease, there was reluctance to dismiss it totally, as JFB has seen cases of lobar pneumonia apparently due to this organism alone or in combination with other bacteria, especially if the patient was debilitated in one way or another. Apart from the background past histories of the three patients, it was clear from the evidence of other holidaymakers that there had been a tendency by many to overdo things during their stay in Spain, namely, over-exposure to the sun, over-eating, over-drinking etc, which may all have contributed to a poor response to the serious infections acquired. (This situation also applies to the two patients who died in 1977.) Further, in attempting to determine a common aetiology in 1973, attention was focused on the numbers of macrophages in the alveoli in all three cases. At least four explanations for this consistent finding were possible: (a) macrophages occur in large numbers at the onset of resolution of lobar pneumonia, (b) macrophages tend to predominate in klebsiella pneumonia (obsolete term Friedlander's pneumonia), which two of the three patients might have had, (c) macrophages tend to predominate when the patient's neutrophil polymorph response is suboptimal due either to the overwhelming nature of the infection, to some primary defect of the haemopoietic system such as agranulocytosis, or to some toxic factor depressing the neutrophil polymorph response, the commonest being alcohol in western society at the present time, and $(d)$ macrophages tend to be more prominent than usual when the natural response to infection has been modified by effective antibiotic therapy. Each of these situations affected all three patients to a varying degree, and, rather prophetically, the 1973 pathology report ended by stating that 'the true causal organism may not be those listed ...'

Specimens from patient 3 were investigated in depth at that time for a viral basis without success, and a similar study was made of patients 4 and 5 during life in 1977 and post mortem in patient 4, again without success.

In summary, the features are very similar to those encountered by an older generation of pathologists, of untreated or neglected lobar pneumonia. The patients received very intensive treatment, although this proved to be ineffectual because it was given too late and because the wrong antibiotics were used. American experience suggests that erythromycin is the recommended antibiotic (Fraser et al., 1977; Fraser et al., 1978). To have had advanced intuition about this possibility does not imply that these patients would have recovered, because it is clear that American patients who had received this antibiotic have died, and we have knowledge of the death of at least one local case in spite of the correct antibiotic regime (Lees et al., 1977). Histology reveals that patients 1-4 had mixed bacterial flora in their lung alveoli, and the empirical antibiotic therapy could have been partly successful in combating the other organisms although ineffective against the LD agent. Postmortem bacteriology of these cases does not reflect fully the histological situation, and this suggests that some of the organisms seen are dead. Deaths from lobar pneumonia can occur at any stage of the process from onset to resolution. All authors are familiar with double lobar pneumonia, but only three of us have experience of triple lobar pneumonia and only two have seen quintuple pneumonia. If it is accepted that the antibiotic therapy administered both in Spain and at home was ineffective, for unforeseen reasons, then the fact that patients survived for 8-13 days implies that all patients had tremendous stamina because of their ability to keep going and resist admission to hospital in Spain, to see their holiday through to the end, to make (or almost make) a strenuous 
air-trip of roughly four hours together with all the protocol at two airports, and to travel their various ways once having returned to Glasgow.

The pathology of the deaths in the Philadelphia Legionnaire outbreak will be documented shortly (Blackmon et al., 1978) but correspondence between Drs J. A. Blackmon and D. W. Fraser of CDC with JFB indicates that there are basically two different sets of histological features. The predominant one is a necrotising lobar pneumonia, which is interpreted by us to imply a pre-suppurative process, and this is evident in selected areas in all of our cases. The necrosis implicates the alveolar exudate rather than the lung structure in the first instance. The other situation noted by the Americans is prominent hyaline membrane formations. The aetiology of these lesions could be multifactorial but is being ascribed, in most instances, to oxygen toxicity, ie is considered to be iatrogenic at the present time. None of our five patients showed this latter feature, and none is considered to have suffered from oxygen toxicity clinically.

The epidemiological studies (Reid et al., 1978) of the 1973 incident suggested that the infection was acquired in Spain. It is easy to lose sight of the fact that some travellers admitted to being unwell before going on holiday, and indeed patient 2 had had an illness labelled pneumonia one month before leaving Glasgow. As mentioned above (Lees et al., 1977), infection indigenous to the west of Scotland is known, just as indigenous sporadic infection is known in America (Fraser et al., 1977) and in England (Ashford et al., 1977; Macrae and Lewis, 1977).

The experience gained from this series raises a further question. How often do pathologists encounter fatal lobar pneumonia as the primary cause of death, for which no aetiological agent is incriminated after satisfactory back-up investigation, including studies for Mycoplasma pneumoniae and psittacosis? This situation is known to two of us although it is not a common one, and a retrospective study of such cases is now required in the light of the recent knowledge of the LD agent.

In preparing this report we acknowledge the great support we have had from numerous colleagues: the clinicians, Drs J. H. Lawson, Ruchill Hospital, Glasgow, R. S. Walker, Law Hospital, Carluke, and I. Michie, County Hospital, Oban; our bacteriology colleagues, Drs R. J. Fallon, A. Mitchell, and F. Russell; Professor N. R. Grist and Dr G. E. D. Urquhart, Regional Virus Laboratory, Ruchill Hospital, and Dr R. G. Sommerville, Belvidere Hospital; Dr D. Reid, Communicable Diseases
(Scotland) Unit, Ruchill Hospital; and the Procurator Fiscal of Paisley for permission to abstract a report submitted to him by virtue of a Warrant of the Sheriff of Renfrewshire. We thank our medical laboratory technical staffs for the histological preparations and Mr E. McWilliams, FIMLS, for the battery of special staining procedures and for the illustrations. We are grateful also to Mrs L. Gilmour for typing the manuscript. At the Center for Disease Control, Atlanta, Georgia, USA, we acknowledge the outstanding help and cooperation that we have had from Drs J. A. Blackmon, P. S. Brachman, W. Cherry, D. W. Fraser, C. C. Shepard, and T. F. Tsai, each for his own specialist contribution to the investigation of these five Scottish cases at a time when they had much to do in connection with the epidemic in Philadelphia, and the very numerous additional studies that have resulted therefrom.

\section{References}

Ashford, R. F. U., Edmonds, M. E., and Shanson, D. C. (1977). Legionnaires' disease in London (Letter). Lancet, 2, 1364.

Blackmon, J. A., Hicklin, M. D., Chandler, F. W., and the Special Expert Pathology Panel (1978). Legionnaires' disease: pathological and historical aspects of a 'new' disease. Archives of Pathology and Laboratory Medicine, 102, (In press).

Chandler, F. W., Hicklin, M. D., and Blackmon, J. A. (1977). Demonstration of the agent of Legionnaires' disease in tissue. New England Journal of Medicine, 297, 1218-1220.

Dieterle, R. R. (1927). Method for demonstration of Spirochaeta pallida in single microscopic sections. Archives of Neurology and Psychiatry, 18, 73-80.

Fraser, D. W., Tsai, T. R., Orenstein, W., Parkin, W. E., Beecham, J., Sharrar, R. G., Harris, J., Mallison, G. F., Martin, S. M., McDade, J. E., Shepard, C. C., Brachman, P. S., and the Field Investigation Team. (1977). Legionnaires' disease. Description of an epidemic of pneumonia. New England Journal of Medicine, 297, 1189-1197.

Fraser, D. W., Wachsmuth, I. K., Bopp, C., Feeley, J. C., and Tsai, T. F. (1978). Antibiotic treatment of guineapigs infected with agent of Legionnaires' disease. Lancet, 1, 175-178.

Lawson, J. H. (1978). Legionnaires' disease-the Benidorm episode. Scottish Medical Journal, 23, 121-124.

Lawson, J. H., Grist, N. R., Reid, D., and Wilson, T. S. (1977). Legionnaires' disease (Letter). Lancet, 2, 1083.

Lees, A. W., Tyrrell, W. F., and Boyd, J. F. (1977). Legionnaires' disease (Letter). Lancet, $2,1187$.

McDade, J. E., Shepard, C. C., Fraser, D. W., Tsai, T. R., Redus, M. A., Dowdle, W. R., and the Laboratory Investigation Team (1977). Legionnaires' disease. Isolation of a bacterium and demonstration of its role in other respiratory disease. New England Journal of Medicine, 297, 1197-1203. 
Macrae, A. D. (1978). Legionnaires' disease (Letter). British Medical Journal, 1, 176-177.

Macrae, A. D., and Lewis, M. J. (1977). Legionnaires' disease in Nottingham (Letter). Lancet, 2, 1225-1226.

van Orden, A. E., and Greer, P. W. (1977). Modification of the Dieterle spirochaete stain. Journal of Histotechnology, 1, 51-53.

Reid, D., Grist, N. R., and Nájera, R. (1978). Illness associated with 'package tours': a combined SpanishScottish study. Bulletin of the World Health Organization, 56, 117-122.

Requests for reprints to: Dr J. F. Boyd, University Department of Pathology of Infectious Diseases, Brownlee Laboratory, Ruchill Hospital, Glasgow G20 9NB. 